Romero-Rodríguez, S., Moreno-Morilla, C. y García Jiménez, E. (2021). La construcción de las identidades culturales en niñas y niños migrantes: Un enfoque desde la etnografía colaborativa. Revista de Investigación Educativa, 39(2), pp-pp.483-501.

DOI: http://dx.doi.org/10.6018/rie.441411

\title{
La construcción de las identidades culturales en niñas y niños migrantes: Un enfoque desde la etnografía colaborativa
}

\section{The construction of cultural identities in migrant children: An approach based on collaborative ethnography}

\author{
Soledad Romero-Rodríguez*, Celia Moreno-Morilla*ı y Eduardo García-Jiménez* \\ *Universidad de Sevilla
}

\begin{abstract}
Resumen
La construcción de las identidades culturales en niñas y niños migrantes requiere de un conocimiento profundo de las experiencias que se desarrollan en diversos espacios (la escuela, el hogar, la mezquita, las asociaciones vecinales, el centro cívico, etc.). Nuestra investigación reflexiona sobre los procesos de construcción de identidades culturales y la aportación de la etnografía colaborativa en su análisis. La utilización de este enfoque metodológico ha permitido la incorporación de la voz del alumnado migrante de Educación Infantil en la exploración de la construcción de sus identidades. Este artículo plantea como objetivo explorar las posibilidades de la etnografía colaborativa en el estudio de la construcción de identidades culturales mediante el análisis semiótico multimodal. Para ello, se ha utilizado un estudio de casos holístico y técnicas propias del enfoque Mosaic como los mapping, el retrato familiar, el roleplay y el autorretrato. El análisis semiótico social multimodal ha puesto en evidencia la difracción que se produce en los diferentes discursos de las niñas y los niños, evidenciando los conflictos en la construcción de sus identidades entre la cultura de origen y la destino, y ha mostrado cómo se configuran los estereotipos culturales en la etapa de Educación Infantil. Las conclusiones de este estudio subrayan la utilidad de la etnografía colaborativa y el análisis semiótico multimodal para el estudio de la construcción de identidades culturales en la infancia.
\end{abstract}

Correspondencia: Celia Moreno Morilla, cmoreno8@us.es, Universidad de Sevilla. 
Palabras clave: comunicación intercultural; identidad; etnografía; semiótica social; Educación Infantil.

\begin{abstract}
The construction of cultural identities in migrant children requires a deep knowledge of the experiences that take place in different spaces (school, home, mosque, neighborhood associations, civic center, etc.). Our research reflects on the processes for the construction of cultural identities and the contribution of collaborative ethnography in their analysis. The use of this methodological approach has allowed for the incorporation of the voice of migrant Preschoolers in the exploration of the construction of their identities. This article aims to explore the possibilities of collaborative ethnography in the study of the construction of cultural identities through multimodal semiotic analysis. For this, a holistic case study and techniques typical of the Mosaic Approach have been used, such as mapping, family portrait, roleplay and self-portraiture. The multimodal social semiotic analysis has highlighted the diffraction that occurs in the different discourses of girls and boys, highlighting conflicts in the construction of their identities between their culture of origin and culture of destination. It has also shown how cultural stereotypes are already configured from an early age. The conclusions of this study underscore the usefulness of collaborative ethnography and multimodal semiotic analysis for the study of the construction of cultural identities in childhood.

Keywords: Intercultural communication; identity; ethnography; social semiotic; Preschool.
\end{abstract}

\title{
Introducción
}

Las identidades culturales se construyen en torno a un conjunto de dimensiones (edad, género, religión, clase social, etnicidad, etc.) que interactúan entre sí. No existe una dimensión unívoca que sea más sobresaliente que las demás, sino una forma única y personal en la que interactúan dichas dimensiones a través de las experiencias vitales que se desarrollan en un eje de privilegio o desventaja social (Arthur, 2019). Sin embargo, algunos aspectos de las identidades culturales pueden ser enfatizados a través de las etiquetas que asignan las otras personas y que pueden llevar a situaciones de ventaja o desventaja social (Arthur, 2017). Desde esta perspectiva, es importante considerar el análisis de las identidades culturales atendiendo a las intersecciones que se producen en los diferentes espacios y situaciones de experiencia vital (Kang, Callaghan y Anne, 2015).

Las identidades culturales comienzan a desarrollarse en la infancia y se manifiesta a través de experiencias vitales que tienen lugar en diferentes espacios. La escuela es un catalizador en la construcción de dicha identidad(es) cultural(es) en la medida que esta se negocia entre alumnado y profesorado. El continuo proceso de negociación de la identidad es alimentado a través de experiencias sociales, donde las analogías y diferencias físicas, lingüísticas o religiosas, entre otras, configuran las representaciones y los estereotipos culturales (Scollon, Scollon y Jones, 2012). Estos elementos se entrelazan en la infancia como capítulos de un relato personal que configura la construcción de la identidad cultural. La identidad cultural es dinámica y conecta no 
sólo elementos emocionales y personales, sino secuencias de relatos heredados de la cultural patrimonial (Jovchelovitch, 2007).

El estudio actual de la infancia plantea la necesidad de conocer la manera en que se desarrollan las identidades culturales a través de los significados que adquieren las distintas representaciones sociales, y se cuestiona el modo en que se configuran los estereotipos culturales, de manera unidireccional o bidireccional (interacción de la cultura nacional del país receptor y/o de la cultura patrimonial).

\section{La etnografía colaborativa}

El estudio de las identidades culturales en la infancia supone todo un reto dadas las características de la edad y sus limitaciones para expresar pensamientos y emociones. El empleo de una perspectiva etnográfica permite afrontar dicho reto desde el conocimiento de la cultura en la que se genera dicha comunicación. Esta perspectiva supone ir más allá del mero empleo de métodos y técnicas habitualmente asociados con el trabajo de campo e implica estudiar las prácticas culturales de un grupo social desde las teorías y los métodos propios de la antropología o la sociología (Green y Bloome, 1997).

Cuando la comunidad objeto de estudio es la escolar, un enfoque etnográfico permite abordar la comunicación intercultural desde el conocimiento de la vida cultural de dicha comunidad (Bloome, 2012). Para ello, el equipo de investigación permanece un largo periodo de tiempo en la escuela y observa numerosas situaciones comunicativas en las que se hacen patentes las identidades culturales de los miembros de la comunidad escolar. No obstante, en un enfoque etnográfico tradicional, la perspectiva del personal investigador es la que predomina en la interpretación del significado de la comunicación intercultural y en la identificación de las identidades culturales, sin olvidar que el personal investigador se posiciona como una persona adulta con formación y experiencia y la persona investigada como alguien que carece de ellas.

La construcción de una etnografía colaborativa permite abordar la comunicación intercultural, pero enfatizando que todas las personas participantes poseen formas válidas de conocimiento y experiencia. El personal investigador trabaja junto con las personas participantes en la investigación a fin de coproducir conocimientos que sean mutuamente accesibles y pertinentes (Haviland, 2017, Cambpbell y Lassiter, 2010, 2014). Este tipo de etnografía nació como reacción a la "perspectiva colonialista" de las investigaciones etnográficas, donde los etnógrafos construían sus reflexiones sobre una comunidad indígena. La etnografía colaborativa cuestiona la perspectiva del personal investigador y su conocimiento anterior sobre un tema concreto y da prioridad a las personas participantes, sus experiencias y conocimientos (Campbell, 2018). Como consecuencia de la aplicación de este enfoque, no siempre es posible diferenciar el conocimiento generado por el personal investigador y por las demás personas participantes (Campbell et al., 2018).

El desarrollo de la etnografía colaborativa enfatiza la colaboración personal investigador-participante en todo el proceso de investigación desde el diseño, a la recogida y la interpretación de los datos (Lassiter, 2005). Esta colaboración supone equidad, pero no igualdad entre personas co-investigadoras, de modo que son posibles diferentes roles en función de la dedicación, los intereses, las habilidades o la confianza en la 
investigación que tienen las personas implicadas. En todo caso, se promueve el respeto a la contribución de los demás (Pushor, 2008).

El acercamiento a la perspectiva de la infancia en un contexto escolar intercultural plantea un nuevo reto relacionado con las diferencias de estatus entre personal investigador y participantes. La etnografía colaborativa sigue un modelo side-by-side, en lugar de top-down, que permite cambiar el foco desde una -investigación sobre las personas participantes - a una "investigación con las personas participantes"(Pushor, 2008).La incorporación de los niños en el mismo plano que el equipo investigador permite oír su voz como parte de la creación de conocimiento sobre la comunicación intercultural en el aula (Rappaport, 2008) y como un modo de comprender la manera en la que los miembros de la comunidad interaccionan con su entorno (Pahl, 2019).

\section{El MosaicApproach}

El desarrollo de la etnografía colaborativa aplicada específicamente a la infancia nació en los años ochenta con el fin de escuchar la voz de las niñas y los niños e incorporar su punto de vista sobre el entorno social y cultural (O'Kane, 2000). La publicación del libro Beyondlistening. Children'sperspectivesonearly Childhood services (Clark et al.,2005) le dio un nuevo impulso, así como la difusión del MosaicApproach que desarrolla una multimetodología de trabajo con la infancia basada en la fotografía, los paseos etnográficos o la elaboración de mapping (Clark y Moss, 2011) y promueve la sistematización de las técnicas de investigación en esta etapa(Harcourt, 2011).

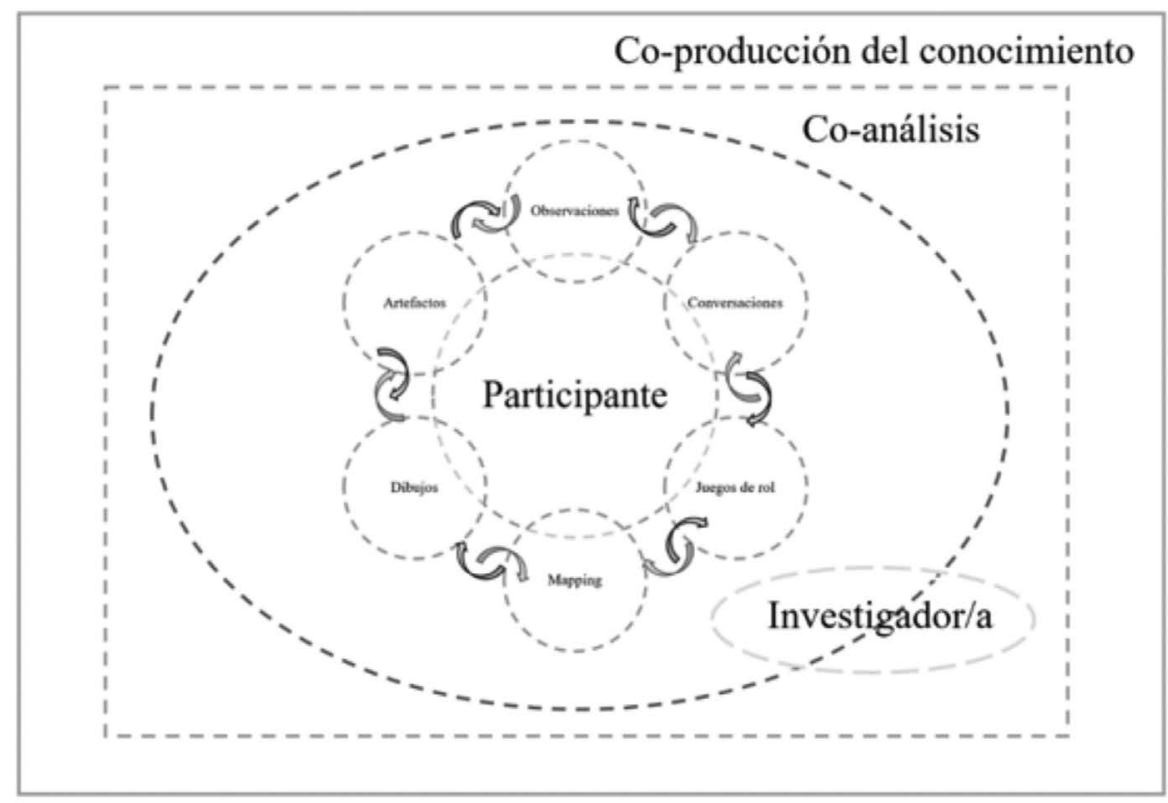

Figura 1. Técnicas de investigación incorporadas en MosaicApproach 
El MosaicApproach desarrollado por Clark (2011a) presenta un enfoque multimetodológico y participativo centrado en las experiencias de las niñas y los niños en las prácticas que desarrollan en su vida diaria (Figura 1). Hacer oír sus voces en un contexto intercultural implica acercarse a su realidad a partir de las diversas estrategias de comunicación que desarrollan. Las identidades culturales toman forma tanto en el discurso verbal como visual o sonoro de las niñas y los niños de modo que, para comprenderlo, es necesario emplear técnicas como la observación (Elfer, 2012) junto a conversaciones breves (child conferencing), juegos de rol (Eide y Winger, 2005), mapping (Clark, 2011a), autorretratos o retratos de familia (Driscoll y Rudge, 2005; Pahl y Rowsell, 2010). A través de esas técnicas es posible construir una imagen de la comunicación intercultural las experiencias de la infancia en el contexto escolar (Clark y Moss, 2011), reflejar sus estereotipos e identidades, así como la manera en la que construyen el conocimiento (Rasool, 2017).

\section{El análisis multimodal y la construcción del conocimiento}

La etnografía ha evolucionado en su comprensión de una cultura mediante la incorporación del conocimiento que proporcionan los sentidos del olfato, el tacto y el oído, situado en un plano de igualdad con el conocimiento generado por los discursos verbal y visual. Algunas autoras (Pink, 2015) han acuñado el término etnografía sensorial para referirse a este modo de acercamiento multimodal a la cultura, mientras que otros han propiciado un acercamiento entre multimodalidad y etnografía (Dicks et al., 2011).

Los discursos multimodales han existido en contextos muy diversos desde el nacimiento de la escritura. Sin embargo, estos discursos han sido abordados desde disciplinas cuyas perspectivas eran monomodales: el discurso verbal, desde la Lingüística; el discurso visual, desde la Historia del Arte; y la música, desde la Musicología (Kress y Leeuwen, 2001). La aceptación de un discurso multimodal en toda su complejidad plantea un problema epistemológico, ya que el análisis se ha de realizar de forma complementaria entre códigos comunicativos verbales, visuales, olfativos, sonoros o táctiles.

La comprensión del significado de la comunicación entre culturas, desde los distintos modos comunicativos presentes en un texto, requiere desarrollar nuevas habilidades de carácter multimodal que hagan posible el diálogo en un mismo plano de diferentes gramáticas (verbal/temporal, visual/espacial, auditiva/temporal). Estas gramáticas configuran un marco teórico semiótico único, que es distinto a los utilizados desde una perspectiva monomodal (Halliday, 1994; O®Toole, 1994). Así, la interpretación del significado del discurso de una niña que ha mezclado conceptos, con olores, colores o sonidos al describir los rasgos de su cultura de origen, requiere activar a un tiempo diferentes lógicas o gramáticas que están en interacción en su discurso.

La etnografía colaborativa plantea que la construcción del conocimiento debe realizarse a partir de la interacción entre participantes y personal investigador. No obstante, cuando fruto de dicha interacción se generan discursos multimodales culturalmente situados, la interpretación de su significado no puede ser neutral. En consecuencia, se requiere adoptar perspectivas de análisis que respeten la naturaleza de dichos discursos y atiendan al contexto en el que se producen. La Semiótica social desarrollada por Halliday (1978), Hodge y Kress (1988) y van Leeuwen (2005), aplicada 
al análisis del discurso multimodal por Fairclough (2003) o Bezemer y Kress (2016), puede ser una alternativa metodológica adecuada por varias razones. En primer lugar, la Semiótica social considera que la construcción del significado (representaciones y estereotipos) no es neutra sino ideológica. En segundo lugar, presta especial atención a los sentidos del discurso (denotación/connotación, metonímico y sinecdótico) y al empleo por los grupos de los códigos dominantes y los sistemas de referencia cultural. En este sentido, resulta especialmente útil para comprender la comunicación que se genera entre culturas que conviven en una misma comunidad como la escuela. Los trabajos de Flewitt (2011) y de Clark (2011) sobre el análisis multimodal del mapping, y de Rowsell (2011) sobre los artefactos, han reforzado la línea argumental de Kress (2011) que sustenta que la investigación etnográfica está ligada en la actualidad a la teoría de la Semiótica social multimodal.

\section{Método}

Esta investigación sigue un enfoque de etnografía colaborativa (Lassiter, 2005) que se basa en el modelo MosaicApproach (Clark y Moss, 2011). Desde este enfoque, las niñas y los niños han adoptado el papel de personal etnógrafo durante la recogida de información, la construcción de evidencias y su posterior co-análisis (Pahl y Pool, 2011).

El diseño seguido se corresponde con un estudio de casos holístico múltiple, tipo 3 (Yin, 2014). Está centrado en una unidad de análisis y permite la replicación y el contraste teórico entre los casos vinculados a diferentes orígenes y/o contextos culturales; cada caso está representado por una niña o un niño en situación de migración.

\section{Objetivos}

Los objetivos de la investigación son:

1. Explorar las posibilidades de la etnografía colaborativa en el estudio de la construcción de identidades culturales.

2. Analizar la construcción de identidades culturales en niñas y niños migrantes mediante el análisis semiótico multimodal.

\section{Población Muestra}

Esta investigación ha contado con la participación de 2 alumnos y 2 alumnas en situación de migración de 5 años de edad 3 personas investigadoras. Todas las personas participantes $(n=7)$ han colaborado en el proceso de recogida y análisis de la información. Los niños están escolarizados en un centro situado en un contexto urbano de exclusión social, considerado Centro de Difícil Desempeño, en el que están matriculados casi 400 alumnos de 3 a 12 años de 33 nacionalidades diferentes.

La selección de participantes se realizó mediante un tipo de muestreo deliberado, denominado de selección de casos críticos (Patton, 2002), que busca la máxima variación en la muestra a partir de un pequeño número de casos. Este tipo de selección nos 
permitió recoger la diversidad de situaciones diarias de comunicación intercultural presentes en el aula (Tabla 1). Los criterios de selección fueron: a) que sus familias estuvieran en situación de migración; b) de países de diferente procedencia; $c$ ) nacidos y no nacidos en España; d) número similar de niños y niñas; y e) interés mostrado por las familias para colaborar en la investigación.

Todas las personas participantes en la investigación eran menores de edad, por lo que sus padres o tutores legales fueron informados sobre la naturaleza del estudio y sus condiciones. La participación fue voluntaria y siguió las reglas de consentimiento informado, que restringe el uso de la información únicamente a fines de investigación y asegura el anonimato y la confidencialidad. Este trabajo ha seguido la regulación interna en Ciencias Sociales por el Comité Ético de Experimentación de la Universidad de Sevilla.

Tabla1

Descripción de los participantes

\begin{tabular}{ll}
\hline Aim & $\begin{array}{l}\text { Nacido en España. Padres marroquíes. El padre tiene una carnicería y la madre se } \\
\text { encarga de las tareas del hogar. }\end{array}$ \\
\hline Guide & $\begin{array}{l}\text { Nacida en España. Padres chadianos. El padre realiza trabajos temporales y ayuda } \\
\text { en un bar de la zona. La madre no se comunica con fluidez en español y se encarga } \\
\text { de las tareas del hogar. }\end{array}$ \\
\hline Alexander & $\begin{array}{l}\text { Nacido en Portugal. Padres colombianos; se desconocen sus profesiones. La } \\
\text { familia no quiere ofrecer información al respecto. }\end{array}$ \\
\hline Sofía & $\begin{array}{l}\text { Nacida en Venezuela. Refugiada. Padres venezolanos. Vive en España con su } \\
\text { madre, que trabaja en un spa; su padre continúa viviendo en Venezuela. }\end{array}$ \\
\hline
\end{tabular}

\section{Procedimientos de recogida y análisis de datos}

La recogida de información se realizó desde un enfoque inspirado en el MosaicApproach (Clark y Moss, 2011), entre octubre de 2017 y junio de 2018, para poder escuchar las voces de los niños sobre su contexto cultural y vida cotidiana en la escuela, hogar y comunidad. Además de la observación participante y la entrevista en profundidad se utilizaron el mapping, el retrato familiar, el roleplay y el autorretrato. Estos últimos eran parte de la rutina de aula mientras que el mapping y el retrato familiar fueron elaborados en sesiones individuales desarrolladas en la biblioteca del centro; las grabaciones en audio y vídeo de todas las sesiones fueron transcritas y analizadas secuencialmente para favorecer la recogida de nuevos datos tras el análisis.

El análisis del discurso multimodal (Kress, 2010) permitió integrar los aspectos verbales, visuales y sonoros hasta obtener una comprensión más holística sobre el proceso de construcción de las identidades culturales, así como la identificación de estereotipos (Pink 2015; Rose, 2016; Bateman, Wildfeuer y Hiippala, 2017; Ledin y Machin (2018). La Tabla 2 recoge la frecuencia o duración de las evidencias recogidas. 
Tabla2

Evidencias recogidas

\begin{tabular}{lll}
\hline Medio/artefactos & Foco & Frecuencia/tiempo \\
\hline Fotografías & Barrio & 97 \\
& Colegio & 98 \\
& Redes Sociales & 5 \\
Vídeos & Casos & 851 \\
Audios & & $37 \mathrm{~h} \mathrm{38}{ }^{\prime}$ 49', \\
Producciones & & $43 \mathrm{~h} 38^{\prime} 49^{\prime \prime}$ \\
Informes Séneca (Administración) & & 582 \\
Informes psicopedagógicos & & 49 \\
Fichas de alfabetización & & 9 \\
Informes de evaluación & & 87 \\
Informes de evaluación de grupo & Casos & 46 \\
Informes de asistencia a clase & & 2 \\
Informes globales por materia & & 10 \\
Entrevistas en profundidad & & 39 \\
Entrevistas autobiográficas & & 6 \\
Mapping & & 49 \\
Autorretratos & & 38 \\
Retrato familiar & & 29 \\
\hline
\end{tabular}

El co-análisis de dicho discurso multimodal permitió a las personas participantes (estudiantes y personal investigador) dialogar sobre los mismos datos para alcanzar una interpretación común (Campbell et al., 2018). El empleo de fotografías, autorretratos o role-play resultó fundamental para que niños tan pequeños compartieran sus interpretaciones con el equipo de investigación, al tiempo que otorgaba credibilidad a los resultados obtenidos (Morrow, 2005).

\section{Resultados}

\section{El mapping como cartografía de las identidades culturales}

El mapping es una técnica que conecta las diferentes experiencias físicas que tienen los niños en sus espacios vitales (Clark, 2011). Esta técnica se utilizó para analizar la construcción de las identidades de los niños en su hogar, en la escuela, en las mezquitas, iglesias, etc. Los niños y el personal investigador hicieron fotografías en estos espacios mediante teléfonos móviles y cámaras. La construcción del mapping fue individual y, durante su elaboración, los niños primero seleccionaron las fotografías más representativas de sus prácticas de comunicación y posteriormente las describieron 
e interpretaron asociando cada espacio con un olor, un sabor, un color y un sonido musical relacionado con sus identidades culturales.
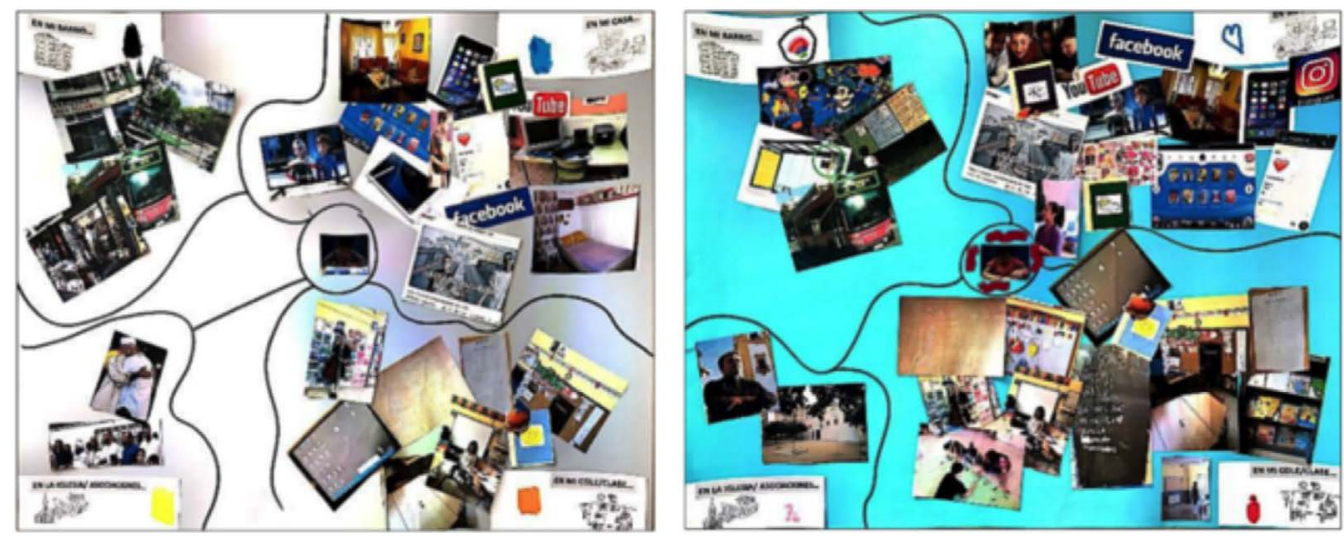

Figura 2. Mapping de Asim (15/06/2018) y Sofía (20/06/2018)

Los mapping recogidos en la Figura 2 muestran la importancia que Asim y Sofía conceden a la comunicación intercultural en su hogar, escuela, barrio o comunidad religiosa. El mapping de Asim da mayor relevancia a los espacios hogar y mezquita frente a la escuela o barrio, de modo que el valor social que atribuye a cada espacio está condicionado principalmente por su identidad cultural y el tipo de prácticas comunicativas desarrolladas en ellos. Asim muestra preferencia por personas de procedencia marroquí ("[Prefiero estar] con gente de Marruecos porque son amigos") que utilizan su lengua patrimonial "[Prefiero] hablar en marroquí"). En este sentido, Asim otorga un papel relevante al imán de la mezquita de su barrio, quien posibilita el acceso a su lengua patrimonial ("Me encanta la mezquita [ ]. Es el profesor que me enseña árabe, hablo árabe con mi padre y con mi madre. [Prefiero hablar] en árabe"). En su interpretación del mapping Asim revela un conflicto cultural ("[He nacido] en España. [Soy] de Marruecos") y un rechazo hacia la nacionalidad española ("No me gusta la bandera de España. [La de Marruecos] tiene una estrella verde bonita y la bandera roja"). El análisis del discurso de Asim refleja el uso extensivo de afirmaciones evaluativas con verbos de afectividad. Estas valoraciones son positivas cuando Asim alude a elementos relacionados con la cultura marroquí (p.e. "Me encanta"; "me gusta"; "es bonita"; etc.) y van acompañadas de elementos de la comunicación no verbal que indican admiración, ilusión, deseo o anhelo. Por el contrario, Asim utiliza verbos de afectividad (en construcciones negativas) para referirse al rechazo de los aspectos culturales identificados con España ("Me aburro"; "no me gusta nada", etc.). Dicha actitud es reforzada por gestos de desconfianza, incertidumbre e inquietud. Otro ejemplo del conflicto en la comunicación intercultural de Amín se evidencia en los colores que asigna a su barrio (negro) y a su casa (azul); el color negro está relacionado con su insatisfacción por vivir en España mientras que el color azul es representativo de su casa de Marruecos, pintada de ese color. En resumen, las representaciones que construye Asim subrayan 
el carácter excluyente de los estereotipos marroquí y español, que alimenta el conflicto de su comunicación intercultural en la escuela.

El análisis del mapping de Sofía plantea otro tipo de conflictos. Sofía señala la necesidad de comprender los motivos que llevaron a parte de su familia a abandonar Venezuela. El mapping de Sofía destaca la importancia de la escuela como espacio seguro y de confianza ("Me gusta este cole porque somos felices"). Por otra parte, Sofía identifica su barrio con un arcoíris de colores, expresión de alegría y bienestar, rasgos que atribuye a España ("[Me gusta más España] porque tiene más comida y la gente vive feliz aquí"), donde prefiere vivir, en oposición a Venezuela, que identifica con la escasez de alimentos ("A veces, a veces, llega la comida, pero cuando queremos comer "toas" las cosas valen mucho"), la oscuridad ("Porque es un poco, es un poco oscurita") y la infelicidad ("Nunca casi [es feliz la gente allí]”). El análisis multimodal del discurso de Sofía recoge gestos que aluden a una mezcla de tristeza, nerviosismo, melancolía y añoranza (ojos entornados, mirada perdida, sonrisa forzada, movimiento rítmico de brazos). Sofía utiliza afirmaciones evaluativas negativas relacionadas con su país de origen ("tonto", "oscurito", "feo", etc.). Algunas de estas afirmaciones tienen un marcado carácter moral ("[Los niños] tienen ropa vieja y sucia, porque los padres son también sucios") y evidencian sus expectativas. Sofía relaciona Venezuela con el olor a tierra ("Como hay tanta tierra, todo se revuelca. ¡Ach! No me gusta cómo huele [Venezuela]) y acompaña su explicación con un gesto de asco. En cambio, cuando Sofía relaciona España con un olor expresa “España me huele a flores. Me gusta mucho España, es bonita", con gesto de dulzura y felicidad. Este rechazo a su país de origen no oculta el conflicto que supone integrar en sus identidades culturales elementos propiamente patrimoniales (" $\mathrm{C}$ : ¿Y te quedarías o te volverías a Venezuela?; S: [Duda antes de contestar] No sé, como quiero a los dos coles. En Venezuela no hay instrumentos [musicales], solamente hay guitarras. Mi profesor también tiene guitarras, me gustan como suenan. En Venezuela [también] cantamos llaneras, y eso [ ]. Un viejo que se parece a mi abuelo canta eso [aquí], y me gusta").

\section{El retrato familiar: la comunicación en la cultura patrimonial}

El retrato de las familias ha sido utilizado como técnica proyectiva a través de la que los niños han representado sus vínculos familiares. Su interés estriba en la información que aporta sobre la comunicación intercultural en el entorno familiar. El retrato de la familia de Asim (Figura 3) contrasta con su discurso. Asim dibuja primero a su padre indicando "[Me quiero parecer] a papá, pero, después, incluye en el dibujo a su tío que vive en Francia y que es un agente alfabetizador y un transmisor de la cultura marroquí ("[Mi tito] me enseña a leer y me cuenta historias de Marruecos, y me pone vídeos de Marruecos"). La importancia de su tío se refleja en su tamaño con relación al de su padre. Después Asim dibuja a su madre, el dibujo de mayor tamaño y que ocupa la posición central en el retrato. Asim vive un verdadero conflicto cultural provocado por la importancia que concede a su madre y el valor social de la mujer en la cultura marroquí ("[Los hombres] son más importantes que las mujeres, me lo dice mi tío“]. Por último, Asim ubica el dibujo de su familia en Marruecos, y la representa con 
indumentaria marroquí. Su retrato familiar muestra cómo construye su identidad a partir de la cultura patrimonial y en conflicto con la cultura representada por la escuela.

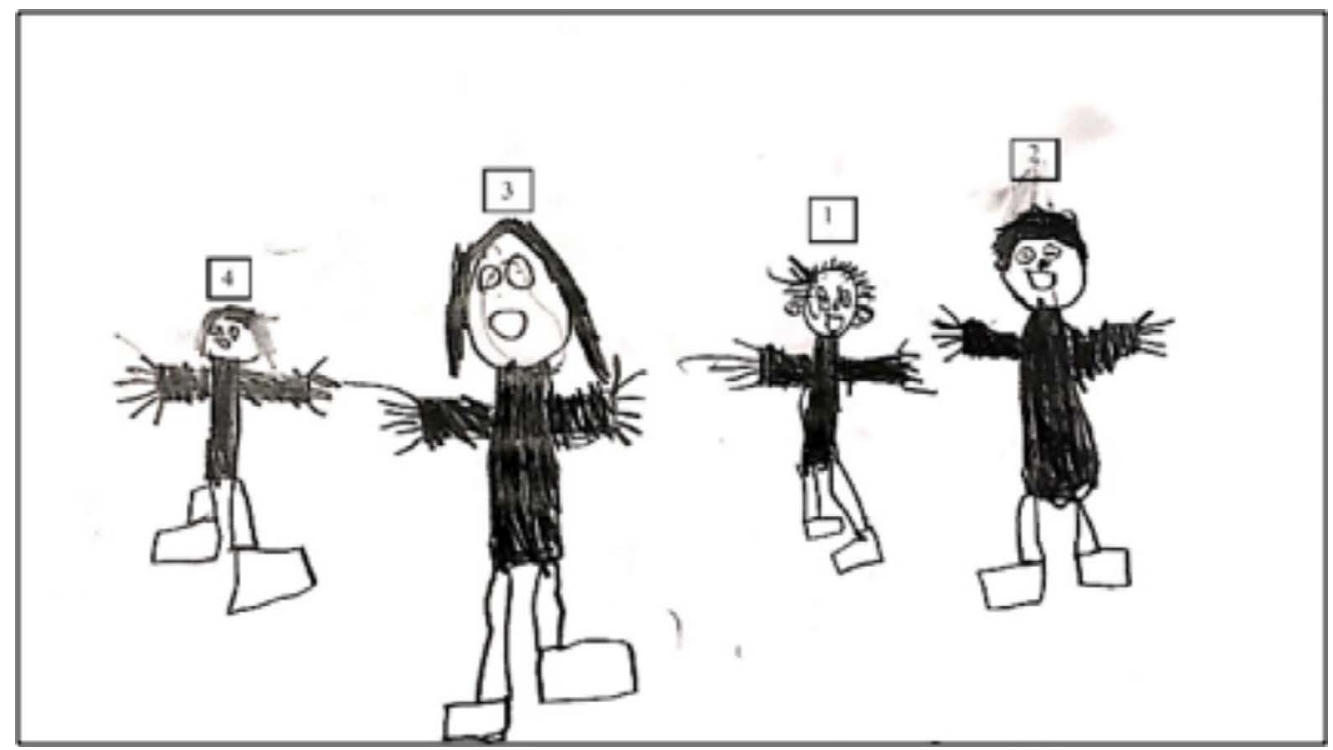

Nota. Asim (4), madre (3), padre (1), tío de Asim (2).

Figura 3. Retrato familiar de Asim (22/03/18)

El retrato familiar de Guida (Figura 4) contrasta con el de Asim. Mientras el coanálisis de Asim refleja una difracción entre su discurso verbal y su retrato familiar, el de Guida busca la coherencia entre ambos discursos. El papel de Guida en su dibujo es central; se dibuja en primer lugar y se representa como agente mediador de la comunicación intercultural entre su familia y el entorno escolar ("Mi mamá no sabe leer español, y yo le estoy ayudando, eso es lo más importante que tiene mi mamá, ella quiere aprender. Pero yo, mi cerebro tiene que saber más todavía para enseñarle muy bien"). Guida dibuja a su madre en segundo lugar y percibe la vulnerabilidad social de su familia representada en el rol de su padre, a quien dibuja en tercer lugar. El padre de Guida aparece distanciado del núcleo compuesto por su madre, su hermano y ella, representado mediante una "casa" que refleja uno de sus múltiples lugares de trabajo ("[Mi papá] trabaja por la mañana, por la tarde y por la noche trabaja mucho y mucho y mucho y viene casi desmayado [ ]. Tiene dos jefes [ ]“). Guida es consciente de la precariedad e inestabilidad de la situación laboral de su padre por ser inmigrante ("Y mi papá está consiguiendo dinero, pero ¿sabes lo más horrible que le ha pasado a mi papá? Le han mentido [enfadada]. Tiene muy poco dinero muy poquito, así mira diminuto [haciendo con la mano el gesto de tamaño pequeño]. Lo tiene diminutito [ ]. Le han en-ga-ña-do [enfatizando cada sílaba]“). Guida se siente responsable de la movilidad social ascendente de su familia asumiendo parte del rol que correspondería a las personas adultas. Guida aspira a conseguir el estatus de clase media occidental 
("Quiero estar bien en mi casa, ir al parque, también ir a la casa de mi amiga"), que representa el día a día del grupo de compañeras al que ella desea pertenecer.

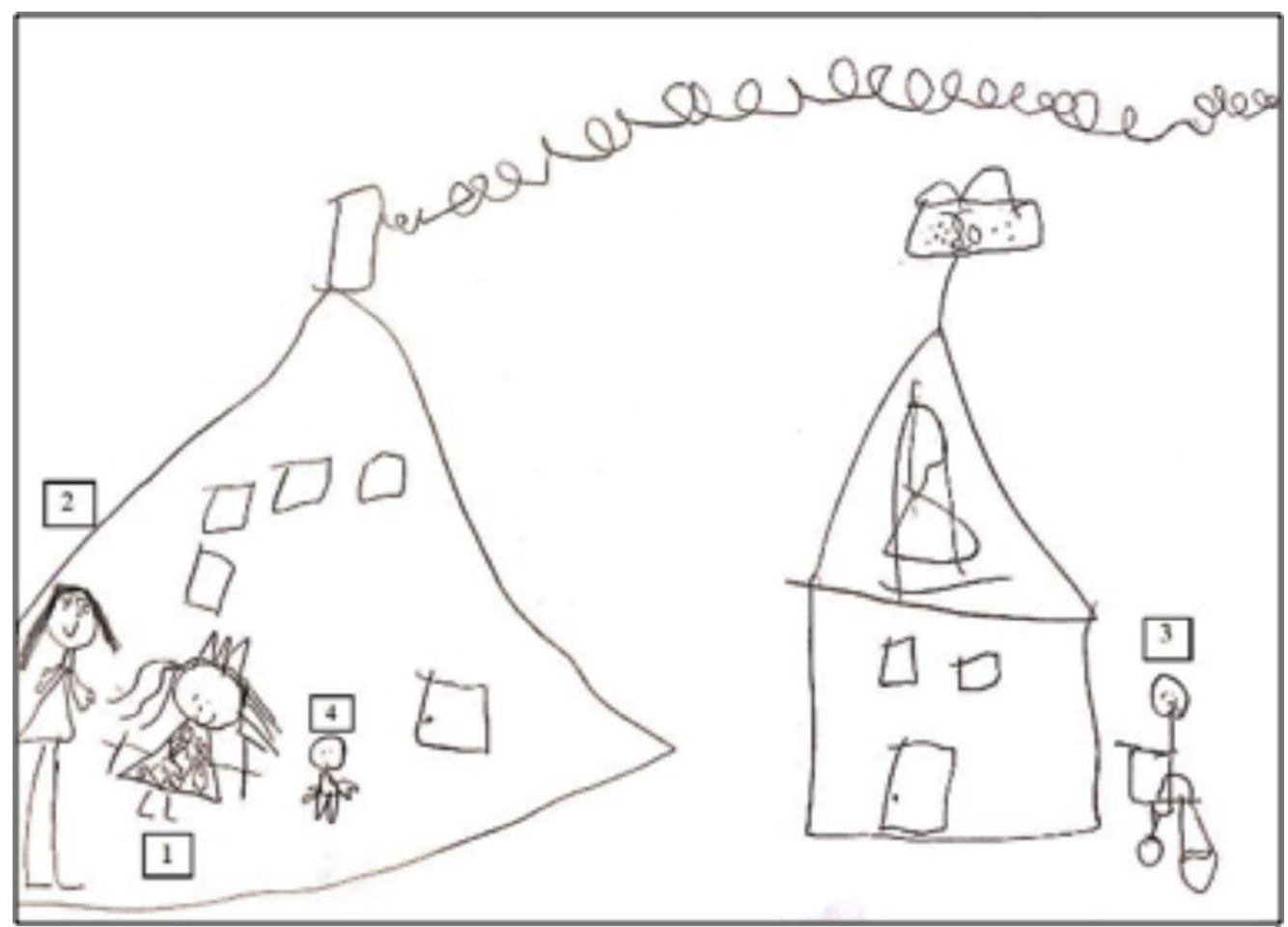

Nota. Guida (1), madre (2), padre (3) y hermano de Guida (4).

Figura 4. Retrato familiar de Guida (22/03/18)

\section{El role-play como representación de la comunicación intercultural en los niños}

La técnica de role-play ha sido utilizada con objeto de relacionar las emociones de los niños y el proceso de construcción de sus identidades. En nuestro estudio, los niños asumieron el rol de "pintores", tomando la identidad artística de Picasso. Concretamente, el role-play se centró en la representación de la "etapa azul" y la "etapa rosa" del pintor. Para ello, el equipo de investigación explicó a los niños cómo Picasso manifestaba su tristeza y alegría a través de la pintura y les presentó cuadros de cada una de las épocas. Por último, se dieron indicaciones sobre las técnicas para pintarlos cuadros. De una parte, los niños debían pensar en algún recuerdo o hecho que les provocara tristeza y utilizar preferentemente tonos azules. Y de otra, los niños debían reflexionar sobre un hecho que les provocara felicidad; en este caso, el color predominante en la pintura debía ser el rosa. Una vez finalizada las pinturas, los niños ponían un título a los cuadros. 


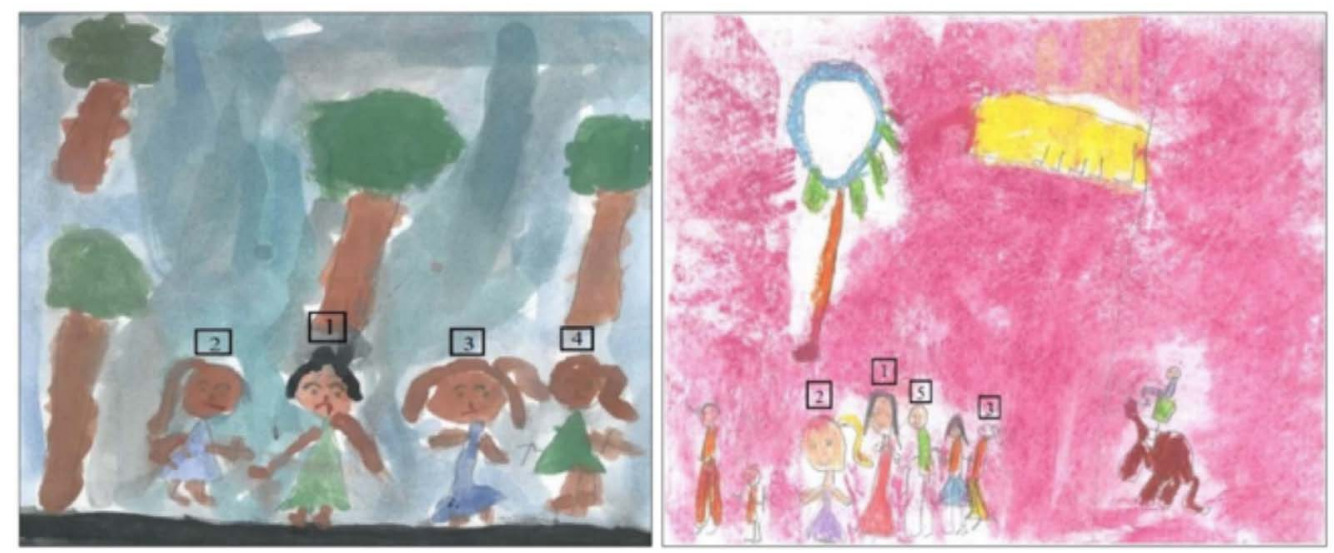

Nota. Guida (1), Paula (2), Nuria (3), Julia (4) (compañeras de Guida), abuelo de Guida (5) (a quien no conoce y vive en África).

Figura 5. La "etapa azul” y la "etapa rosa" de Guida (26/04/18 y 27/04/18, respectivamente)

El cuadro realizado por Guida para representar su "etapa azul" (Figura 5) se titula "Niñas que no me dejan jugar" y, en la descripción de su pintura, escribe: "Estoy triste porque a veces no me dejan jugar con ellas en el patio". Este grupo de compañeras son identificadas por Guida como el prototipo de clase media al que aspira pertenecer. El deseo de comunicarse en un plano de igualdad con sus compañeras le lleva a rechazar su cultura patrimonial ("En ningún motivo me gusta África [], las tonterías esas que matan y eso. No quiero ir allí nunca"). El análisis multimodal y crítico de su discurso hace más evidente el conflicto en la construcción de sus identidades culturales. Guida utiliza afirmaciones evaluativas de modalidad epistémica ("Aquí mismo. Me quedaría aquí mismo"), acompañándolas de un tono de voz y de gestos faciales que indican enfado, rechazo y tristeza. La "etapa rosa" de Guida (Figura 5) muestra una escena de su familia en la Feria de Sevilla. Guida incluye en su pintura la representación de varios miembros de su familia extensa (p.e. su abuelo y un camello). El co-análisis de ambas pinturas realizado con Guida evidencia el conflicto que se genera en la construcción de su identidad en la escuela, donde su cultura patrimonial no tenía cabida.

\section{El autorretrato como representación de identidades étnicas y culturales}

La técnica del autorretrato se utilizó para que las niñas y los niños exploraran la representación de sus identidades étnicas y culturales. A cada niño se le proporcionó un espejo y una camiseta de color blanco. El co-análisis se realizó sobre el autorretrato pintado en la camiseta y se centró en los colores utilizados y los sentimientos que despertaban; además se les pidió que se identificaran con un olor, un color, un sabor $\mathrm{y}$ un sonido musical. 

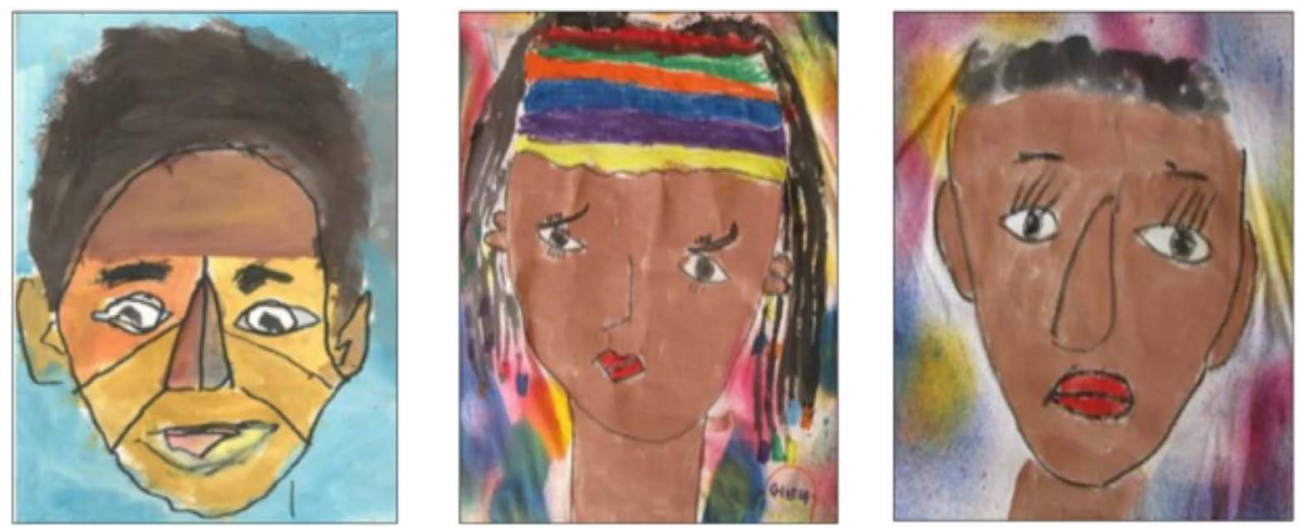

Figura 6. Autorretratos de Asim, Guida y Alexander (entre 10/05/18 y 31/05/18)

Los niños manifestaron una conciencia clara de sus características y rasgos físicos (color de piel, tipo de pelo, etc.). Estas características fueron identificadas con rasgos étnicos atribuidos socialmente a sus países de origen. Asim, Guida y Alexander (Figura 6) representaron estereotipos ("Como soy yo") que, a través de un análisis semiótico multimodal, nos permitieron acercarnos a sus identidades. Asim relaciona su imagen con Marruecos y anhela haber nacido en Marruecos ("[Yo he nacido] en Ma en España, soy de Marruecos"); cuando habla articula con claridad y eleva el volumen cuando describe sus rasgos físicos. En cambio, Guida y Alexander generan un diálogo comparativo, que en ocasiones los lleva a describirse desde la diferencia. Ambos perciben estas diferencias como una dificultad en la comunicación intercultural en la escuela. Alexander manifiesta "[No soy como tú], porque mi cara es marrón [ ], yo soy de otro país". La conciencia de los estereotipos étnicos parece plantearle ciertos problemas emocionales a Alexander, lo que se refleja en el análisis multimodal de su discurso (p.e. mantiene la cabeza inclinada hacia abajo, se muestra inquieto y sus gestos faciales y la dirección de la mirada expresan inseguridad y tristeza). Por otro lado, Guida reconoce su color de piel y utiliza el marrón para pintarse. Cuando hace referencia en la conversación a los estereotipos étnicos, Guida utiliza una modalidad discursiva de valoración asumida ("[He elegido el marrón] porque soy así"), mientras subraya dicha afirmación con determinados rasgos paratextuales (aumento del ritmo e intensidad). Los discursos verbal y no verbal de Guida expresan con naturalidad la aceptación de los estereotipos étnicos que son parte de su identidad. No obstante, para Guida su identidad es un obstáculo para pertenecer al grupo sociocultural que es su referente en la escuela. Ello genera un conflicto en la construcción de su identidad que la llevan a representarse ocasionalmente en sus pinturas como una niña de piel blanca, pelo liso, vestida de princesa y rodeada de personas que comparten estos rasgos.

\section{Discusión y conclusiones}

Nuestro trabajo ha pretendido explorar las posibilidades de la etnografía colaborativa para investigar un problema tan complejo como la comunicación intercultural en 
un colectivo igualmente complejo, representado por los niños de Educación infantil. Esta propuesta metodológica ha permitido el acercamiento a dicho tópico desde una perspectiva diferente, que ha privilegiado la escucha de la voz de los niños y su experiencia en un espacio escolar intercultural. Los resultados de esta investigación han mostrado cómo la etnografía colaborativa (Lassiter, 2005) y el MosaicApproach (Clark y Moss, 2011), junto al análisis semiótico multimodal, son excelentes herramientas para acceder al estudio de la comunicación intercultural en Educación infantil.

La etnografía colaborativa ha facilitado que las niñas y los niños se expresen por sí mismos y muestren su(s) identidad(es). Del mismo modo, el co-análisis en el que se apoya la etnografía colaborativa se ha mostrado como una técnica efectiva que hace posible que los propios niños y niñas interpreten el significado de sus experiencias, excluyendo parcialmente las identidades culturales del personal investigador (Campbell, 2018). Durante las sesiones de co-análisis, las niñas y los niños han tenido la oportunidad de compartir los procesos de construcción de sus identidades étnicas y culturales, mientras narraban sus experiencias, como sugieren los trabajos de Gibson (2004) y Meijers y Lengelle (2012).

La investigación colaborativa ha permitido constatar la compleja construcción de los estereotipos e identidades en las aulas de Educación infantil. Este estudio ha abierto la posibilidad de abordar los conflictos en el marco de la construcción de identidades en la escuela desde una nueva perspectiva: la pedagogía colaborativa de Campbell y Lassiter (2010). Su planteamiento supone transformar la etnografía colaborativa en una metodología de aprendizaje donde el conocimiento es construido en las aulas de forma colaborativa y las técnicas utilizadas se convierten en actividades de aula. Así, el personal docente en Educación infantil puede integrar herramientas que incentivan la escucha de las voces de la infancia, especialmente en situaciones complejas como las que se dan en contextos escolares multiculturales y/o en centros denominados "de difícil desempeño". Este planteamiento ha sido llevado a cabo en el proyecto Imagine: Connecting Communities Through Reseach, desarrollado en un contexto de vulnerabilidad social (Banks et al., 2019; Campbell et al., 2018; Facer y Pahl, 2017), donde era preciso incorporar metodologías que fomentasen la equidad y la justicia social (Kassan et al., 2018).

La incorporación del análisis del discurso multimodal de la construcción de identidades culturales ha sido crucial en esta investigación. La información recogida a partir de formas de expresión oral, escrita, visual y corporal ha sido posible integrarla gracias a la Semiótica social. Este enfoque nos ha permitido determinar el valor social de los distintos modos de comunicación y profundizar en la construcción de las identidades en la infancia. El uso de metodologías visuales y artísticas de comunicación ha favorecido que los niños se sientan escuchados y colaboren activamente en la construcción de un diálogo significativo para ellos (Byrne et al., 2016; Taylor y Savickas, 2016). El mapping, el retrato familiar, el role-play o el autorretrato han ayudado a los niños a evocar informaciones, sentimientos y recuerdos que podrían no haber surgido, o ser difíciles de describir a través de un discurso verbal.

La escuela tiene la oportunidad de abordar el proceso de construcción de las identidades culturales en el aula a través de la multimodalidad del discurso. La asunción de esta perspectiva comunicativa permitiría aprovechar el potencial de todos los artefactos creados por los niños dentro y fuera de la escuela como parte de la comu- 
nicación intercultural. Así, el desarrollo de las identidades étnica y cultural se haría más visible y comprensible para los demás y contribuiría a reconfigurar los valores sociales atribuidos a los estereotipos (Dockett et al., 2017).

\section{Referencias}

Arthur, N. (2017). Constructivist approaches to career counselling: A culture-infused approach. In M. McMahon (Ed.). Career counselling: constructivist approaches. (pp. 54-64). Routledge.

Arthur, N. (2019). Career developmet theory and practice. A culture-infused perspective. In N. Arthur y M. McMahon (Ed.). Contemporary theories of career development (pp. 180-194). Routledge.

Banks, S., Hart, A., Pahl, K. y Ward, P. (Eds.) (2019). Co-producing research: A community development approach. Policy Press/University of Bristol.

Banks, S. Hart, A., Pahl, K. y Ward, P. (2019). Co-producing research: a community development approach. En S. Banks, A. Hart, K. Pahl y P. Ward (Eds.), Co-producing research. A community development approach (pp. 1-18). Policy Press/University of Bristol.

Bateman, J., Wildfeuer, J. y Hiippala, T. (2017).Multimodality. Foundations, research and analysis. A problem-oriented introduction. De Gruyter Mouton.

Bell, D., y Pahl, K. (2018). Co-production: Towards a utopian approach. International Journal of Social. Research Methodology, 21, 105-117. https://doi.org/10.1080/136455 79.2017.1348581

Bezemer, J. y Kress, G. (2016). Multimodality, learning and communication. A social semiotic frame. Routledge.

Bloome, D. (2012). Classroom ethnography. En M. Grenfell, D. Bloome, C. Hardy, K. Pahl, J. Rowsell y B. Street, Language, ethnography, and education. Bridging new literacy studies and Borudieu (pp. 7-26). Routledge.

Blustein, D. L., Franklin, A. J., Makiwane, M., y Gutowski, E. (2017). Unemployment in South Africa. In G. B. Stead, y M. B. Watson (Eds.). Career psychology in the South African context (p. 195-208). Van Schaik.

Byrne, E., Daykin, N. y Coad, J. (2016). Participatory Photography in Qualitative Research: A Methodological Review. Visual Methodologies, 4 (2), 1-12. http://dx.doi. org/10.7331/vm.v4i2.66

Campbell, E. (2018). Methodology: an introduction. In E. Campbell, K. Pahl, E. Pente, y Z. Rasool, Re-Imagining contested communities. Connecting Rotherham through research (pp. 87-90). Policy Press/University of Bristol.

Campbell, E. y Lassiter, L.E. (2010). From collaborative ethnography to collaborative pedagogy: Reflections on the other side of Middletown Project and Community-University research partnerships. Anthropology y Education Quarterly, 41(4), 370-385. http://doi.org/10.1111/j.1548-1492.2010.01098.x

Campbell, E. y Lassiter, L.E. (2014). Doing ethnography today. Theories, methods, exercises. Wiley-Blackwell. 
Campbell, E., Lassiter, L.E. y Pahl, K. (2018). Collaborative ethnography in context. In E. Capmbell, K. Pahl, E. Pente, y Z. Rasool (Eds.), Re-Imagining contested communities. Connecting Rotherham through research (pp. 91-106). Policy Press/University of Bristol.

Campbell, E., Pahl, K. Pente, E. y Rasool, Z. (Eds.) (2018). Re-imagining contested communities. Connecting Rotherham through research. Policy Press/University of Bristol.

Clark, A. (2005). Ways of seeing: Using the mosaic approach to listen to young children's perspectives. En A. Clark, P. Moss, y A. Kjørholt (Eds.), Beyond listening: Children's perspectives on early childhood services (pp. 29-49). The Policy Press.

Clark, A. (2011a). Multimodal map making with young children: exploring ethnographic and participatory methods. Qualitative Research, 11(3), 311-330. https://doi. org/10.1177/1468794111400532

Clark, A. (2011b). Breaking methodological boundaries? Exploring visual, participatory methods with adults and young children. European Early Childhood Education Research Journal, 19(3), 321-330. https://doi.org/10.1080/1350293X.2011.597964

Clark, A. y Moss, P. (2011). Listening to young children. The Mosaic approach. NCB.

Clark, A., Kjørholt, A.T. y Moss, P. (Eds.) (2005). Beyond listening. Children's perspectives on early childhood services. Policy Press/University of Bristol.

Dervin, F. (2012). Cultural Identity, Representation and Othering. In J. Jackson (Ed.), The Routledge handbook of language and intercultural communication (pp. 169-180). Routledge.

Dicks, Flewitt, R., Lancaster, L. y Pahl, K. (2011). Multimodality and ethnography: working at the intersection. Qualitative Research, 11(3), 227-237. https://doi. org/10.1177/1468794111400682

Dockett, S., Einarsdottir, J. y Perry, B. (2017). Photo elicitation: reflecting on multiple sites of meaning. International Journal of Early Years Education, 25(3), 225-240. https:// doi.org/10.1080/09669760.2017.1329713

Driscoll, V. y Rudge, C. (2005). Channels for listening to young children and parents. En A. Clark, A.T. Kjørholt y P. Moss (Eds.), Beyond listening. Children's perspectives on early childhood services (pp. 91-110). Policy Press/University of Bristol.

Eide, B.J. y Winger, N. (2005). From the children's point of view: methodological and ethical challenges. En A. Clark, A.T. Kjørholt y P. Moss (Eds.), Beyond listening. Children's perspectives on early childhood services (pp. 71-89). Policy Press/University of Bristol.

Elfer, P. (2012). Psychoanalytic methods of observation as a research tool for exploring young children's nursery experience. International Journal of Social Research Methodology, 15(3), 225-238. https://doi.org/10.1080/13645579.2011.582295

Facer, K. y Pahl, K. (Eds.) (2017). Valuing interdisciplinary collaborative research. Beyond impact. Policy Press/University of Bristol.

Faubion, J.D. y Marcus, G.E. (2009). Fieldwork is not what it used to be: Learning anthropology's method in a time of transition. Cornell University Press.

Flewitt, R. (2011). Bringing ethnography to a multimodal investigation of early literacy in a digital age. Qualitative Research, 11(3), 293-310. https://doi. org/10.1177/1468794111399838

Gibson, P. (2004). Where to from here? A narrative approach to career counselling. Career Development International, 9 (2), 176-189. 
Halliday, M. (1978). Language as Social Semiotic: The Social Interpretation of Language and meaning. Edward Arnold.https://doi.org/10.1108/13620430410526201

Harcourt, D. (2011). An encounter with children: seeking meaning and understanding about childhood. European Early Childhood Education Research Journal, 19(3), 331-343. https://doi.org/10.1080/1350293X.2011.597965

Hodge, R. y Kress, G. (1988). Social Semiotics. Cornell University Press.

Jovchelovitch, S. (2007). Knowledge in context. Routledge.

Kang, H., Callaghan, J. y Anne, M. (2015). An intersectional social capital model of career development for international marriage inmigrants. The Career Development Quarterly, 63, 238-252.https://doi.org/10.1002/cdq.12016

Kassan, A., Goopy, S., Green, A., Arthur, N., Nutter, S., Russell-Mayhew, S., Sesma Vazquez, M. y Silversides, H. (2018). Becoming together: making meaning with newcomers through an arts-based ethnographic research design. Qualitative Research in Psychology,17(2), 294-311.https://doi.org/10.1080/14780887.2018.1442769

Kress, G. (2010) Multimodality. A Social Semiotic Approach to Contemporary Communication. Routledge.

Kress, G. (2011). Partnerships in research: multimodality and ethnography. Qualitative Research, 11(3), 239-260. https://doi.org/10.1177/1468794111399836

Kress, G. y van Leeuwen, T. (2008). Reading images. The grammar of visual design.Routledge.

Lassiter, L. E. (2005). Collaborative Ethnography and Public Anthropology. Current Anthropology, 46(1), 83-97.https://doi.org/10.1086/425658

Ledin, P. y Machin, D. (2018). Doing visual analysis. From theory to practice. Sage.

Meijers, F. y Lengelle, R. (2012). Narratives at work: The development of career identity. British Journal of Guidance and Counselling, 40, 157-176. https://doi.org/10.1080/030 69885.2012.665159

Morrow, S. L. (2005). Quality and trustworthiness in qualitative research and counseling psychology. Journal of Counseling Psychology, 52, 250-260. https://doi.org/10.1037/00220167.52.2.250

O'Kane, C. (2000). The development of participatory techniques: facilitating children's views about decisions which affect them. In P. Christensen y A. James (Eds.), Research with children: perspectives and practice (pp. 136-159). Falmer Press.

Pahl, K. (2019). Re-thinking literacies with communities. Literacy as a Collaborative concept. En M. Grenfell y K. Pahl, Bourdieu, language-based ethnographies and reflexivity. Putting theory into practice (pp. 57-72). Routledge.

Pahl, K. y Rowsell, J. (2010). Artifactural literacies. Every object tells a story. Teacher College Press.

Pahl, K. y Pool, S. (2011). 'Living your life because it's the only life you've got': Participatory research as a site for discovery in a creative project in a primary school. Qualitative Research Journal, 11, 17-37. https://doi.org/10.3316/QRJ1102017

Patton, M.Q. (2002) Qualitative Research and Evaluation Methods (3rd edn). Sage.

Pink, S. (2015). Doing sensory ethnography. Sage.

Pink, S. (2011). Multimodality, multisensoriality and ethnography knowing: social semiotics and the phenomenology of perception. Qualitative Research, 11(3), 261-276. https://doi.org/10.1177/1468794111399835 
Rappaport, J. (2008). Beyond participant observation. Collaborative ethnography as theoretical innovation. Collaborative Anthropologies, 1, 1-31. https://doi.org/10.1353/ cla.0.0014

Rasool, Z. (2017). Collaborative working practices: Imagining better research partnerships. Research for all, 1(2), 310-322. https://doi.org/10.18546/RFA.01.2.08

Rose, G. (2016). Visual methodologies. An introduction to researching with visual materials. Sage.https://doi.org/10.18546/RFA.01.2.08

Rowsell, J. (2011). Carrying my family with me: artifacts as emic perspectives. Qualitative Research, 11(3), 331-346. https://doi.org/10.1177/1468794111399841

Scherr, A. (2007). School and cultural difference. In H. Kotthoff y H. Spencer-Oatey, Handbook of Intercultural Communication (pp. 303-321). Mouton de Gruyter.

Scollon, R. y Scollon, S.W. (2001). Discourse and intercultural communication. In D. Schiffrin, D. Tannen, y H.E. Hamilton (Eds.), The Handbook of Discourse Analysis (pp. 538-547). Blackwell.

Scollon, R., Scollon, S.W. y Jones, R.H. (2012). Intercultural communication. A discourse approach. Wiley-Blackwell.

Street, B. (2010). Adopting an ethnographic perspective in research and pedagogy. En C. Coffin, T. Lillis, y K. O'Halloran (Eds.), Applied Linguistics Methods (pp. 201-215). Routledge.

Taylor, J.M., y Savickas, S. (2016). Narrative career counseling: My career story and pictorial narratives. Journal of Vocational Behavior 97, 68-77. https://doi.org/10.1016/j. jvb.2016.07.010

Van Leeuwen, T. (2005). Introducing Social Semiotics. Routledge

Fecha de recepción: 1 de septiembre de 2020.

Fecha de revisión: 27 de septiembre de 2020.

Fecha de aceptación: 1 de noviembre de 2020. 